\title{
Índices predictores de eventos adversos en el adulto inmunocompetente hospitalizado por neumonía neumocóccica adquirida en la comunidad
}

\author{
Fernando Saldías P. y Orlando Díaz P.
}

\section{Severity scores for predicting clinically relevant outcomes for immunocompetent adult patients hospitalized with community-acquired pneumococcal pneumonia}

Streptococcus pneumoniae is the main cause of community-acquired pneumonia (CAP) in adults. Objectives: To compare accuracy and discriminatory power of three validated rules for predicting clinically relevant adverse outcomes in patients hospitalized with community-acquired pneumococcal pneumonia. Measurements: We prospectively compared the pneumonia severity index (PSI), British Thoracic Society score (CURB-65) and severe CAP score (SCAP) in a cohort of 151 consecutive immunocompetent adult patients hospitalized with pneumococcal pneumonia. Major adverse outcomes were admission to ICU, need for mechanical ventilation, in-hospital complications and 30-day mortality. Mean hospital length of stay (LOS) was also evaluated. The predictive indexes were compared based on sensitivity, specificity, and area under the curve of the receiver operating characteristic. Results: The mean age of 151 immunocompetent adult patients hospitalized with pneumococcal pneumonia was 64 years (range, 16 to 92); $58 \%$ were male, $75 \%$ had comorbidities, $26 \%$ were admitted to the intensive care unit and $9 \%$ needed mechanical ventilation. The rate of all adverse outcomes and hospital LOS increased directly with increasing PSI, CURB-65 and SCAP scores. The three severity scores allowed us to predict the risk of in-hospital complications and 30-day mortality. The PSI score was more sensitive and the SCAP was more specific to predict in-hospital complications and the risk of death. However, the SCAP was more sensitive and specific in predicting the use of mechanical ventilation. Conclusion: The severity scores validated in the literature allow us to predict the risk of complications and death in adult patients hospitalized with pneumococcal pneumonia. Nevertheless, the clinical indexes differ in their sensitivity, specificity and discriminatory power to predict different adverse events.

Key words: Streptococcus pneumoniae, community-acquired pneumonia, severity assessment, mortality, risk classification, prediction rule.

Palabras clave: Streptococcus pneumoniae, neumonía adquirida en la comunidad, evaluación de la gravedad, mortalidad, clasificación de riesgo, regla de predicción.

\section{Introducción}

S treptococcus pneumoniae es el principal agente causal de neumonía adquirida en la comunidad (NAC) en el adulto inmunocompetente ${ }^{1-3}$, con una incidencia estimada de 6-10 casos por 100.000 personasaño ${ }^{4,5}$. La mortalidad de la neumonía neumocóccica es cercana a $5 \%$ y aumenta significativamente en el adulto mayor y el hospedero inmunocomprometido. La morbimortalidad descendió significativamente con la introducción de la antibioterapia ${ }^{6}$; sin embargo, el riesgo de complicaciones y muerte por neumonía varía en la población ${ }^{3}$, dependiendo de la edad del hospedero, su estado inmune, presencia de co-morbilidades, agente etiológico, gravedad y extensión de la infección pulmonar, y grado de adherencia al tratamiento recomendado en las guías clínicas ${ }^{7-9}$.

Una vez establecido el diagnóstico, las guías clínicas de neumonía ${ }^{10-12}$ recomiendan evaluar la gravedad del paciente considerando variables socio-demográficas, de la historia clínica y examen físico, hallazgos radiográficos y de los exámenes de laboratorio. La evaluación de la gravedad permite estimar el riesgo de complicaciones y muerte del paciente con neumonía, decidir la extensión del estudio microbiológico, la necesidad de solicitar exámenes complementarios y el lugar de manejo (ambulatorio o en el hospital), y planificar el tratamiento antimicrobiano empírico, la ruta de administración y su duración ${ }^{12}$.

Con el objeto de sistematizar la evaluación de la gravedad de los pacientes con NAC, se han diseñado y validado en diferentes áreas geográficas diversos índices pronósticos, tales como el índice de gravedad de la neumonía-IGN (en inglés pneumonia severity index -PSI) descrito por Fine y cols ${ }^{13}$, el índice pronóstico de la Sociedad Británica de Tórax modificado por Lim y cols ${ }^{14}$ (CURB-65), y recientemente, el índice de neumonía grave
Pontificia Universidad Católica de Chile, Santiago, Chile.

Facultad de Medicina Departamento de Enfermedades Respiratorias.

Conflictos de interés: Financimiento:

Recibido: 1 de julio de 2010 Aceptado: 20 de mayo de 2011

Correspondencia a: Fernando Saldías Peñafiel fsaldias@med.puc.cl 
adquirida en la comunidad-INGAC (en inglés severe community acquired pneumonia -SCAP) validado en población europea ${ }^{15}$, los cuales son utilizados para estimar la gravedad de los pacientes con neumonía atendidos en el ámbito ambulatorio y decidir el lugar de manejo. Sin embargo, estos índices fueron primariamente diseñados para evaluar el riesgo de muerte de los pacientes con neumonía, y con excepción del INGAC, no se examinaron otros eventos clínicamente relevantes en la evolución, tales como la admisión a UCI, conexión a ventilador mecánico y desarrollo de complicaciones en el hospital (ej.: síndrome coronario agudo, arritmias, shock, empiema).

Por esta razón, en este estudio evaluamos la capacidad discriminativa de tres índices pronósticos (IGN, CURB-65 e INGAC) para predecir eventos adversos clínicamente relevantes (admisión a UCI, uso de ventilación mecánica y complicaciones en el hospital) en pacientes adultos inmunocompetentes hospitalizados por neumonía neumocóccica adquirida en la comunidad.

\section{Pacientes y Método}

Entre el $1^{\circ}$ de enero de 2002 y 31 de diciembre de 2005 se reclutaron prospectivamente 151 pacientes adultos inmunocompetentes hospitalizados por neumonía neumocóccica adquirida en la comunidad, en el Hospital Clínico de la Pontificia Universidad Católica de Chile. Los pacientes tenían 15 años de edad o más y cumplían los criterios diagnósticos de NAC propuestos por Fang y cols ${ }^{16}$. La infección neumocóccica fue confirmada mediante cultivo de expectoración, sangre y/o líquido pleural o antígeno urinario (Binax Inc., Scarborough ME) positivos para S. pneumoniae. Se excluyó del estudio a pacientes bajo 15 años de edad, a los hospitalizados durante el mes previo al ingreso y a aquellos que tenían alguno de los siguientes criterios de inmunosupresión: infección por VIH, neutropenia (menor de $1.000 / \mathrm{mm}^{3}$ ), tratamiento quimioterápico o inmunosupresor en los seis meses previos a la admisión y uso $\geq 20 \mathrm{mg} /$ día de prednisona o su equivalente durante los últimos tres meses. Los investigadores no participaron en el manejo de los pacientes y el estudio fue aprobado por el Comité de Ética de la Institución.

En la admisión al hospital se consignaron las características sociodemográficas, co-morbilidades, cuadro clínico y exámenes de laboratorio que permitieron calcular los tres índices pronósticos examinados: IGN, CURB-65 e INGAC ${ }^{13-15}$. Además, se calculó la variante CRB-65, que a diferencia del CURB-65, no requiere exámenes de laboratorio y puede ser utilizada por los médicos en el ámbito ambulatorio ${ }^{17}$. El compromiso cualitativo de conciencia o confusión mental fue definido por la presencia de desorientación en tiempo, espacio y/o personas que no corresponden a una condición basal conocida o coma.
La descripción de la radiografía de tórax fue realizada por un radiólogo del Departamento de Radiología, quien desconocía el cuadro clínico de los pacientes.

El IGN permite distinguir cinco categorías de riesgo (clases I a V), correspondiendo a las categorías IV-V el mayor riesgo de muerte ${ }^{13}$. En el CURB-65, la categoría de alto riesgo corresponde a los pacientes que tienen puntaje mayor o igual a tres ${ }^{14}$. El CRB-65 define la categoría de riesgo elevado por un puntaje mayor o igual a dos ${ }^{17}$. El INGAC distingue cinco categorías de riesgo (0, 1-9, $10-19,20-29, \geq 30$ puntos) correspondiendo a las dos categorías superiores el mayor riesgo de complicaciones y muerte ${ }^{15}$.

En el hospital se consignaron las siguientes variables: tratamiento antimicrobiano empírico, admisión a UCI y conexión a ventilador mecánico, y desarrollo de las siguientes complicaciones: insuficiencia respiratoria grave $\left(\mathrm{PaO}_{2} / \mathrm{FiO}_{2}<200\right)$, síndrome de distrés respiratorio agudo, arritmias (taquicardia paroxística supraventricular, flutter, fibrilación auricular, taquicardia ventricular), insuficiencia cardíaca congestiva, isquemia miocárdica, accidente vascular encefálico, insuficiencia renal aguda, shock séptico, empiema pleural e infección extra-pulmonar. El tratamiento antimicrobiano fue considerado apropiado si al menos un antimicrobiano era activo contra el microorganismo aislado. Además, se consignó la mortalidad cruda en el hospital y en el seguimiento a 30 días después de la admisión.

\section{Evaluación microbiológica}

Las muestras de sangre fueron inoculadas en botellas BacT/Alert ${ }^{\circledR}$ (Biomerieux) e incubadas por siete días en el sistema automatizado. En las muestras de sangre que hubo desarrollo de microorganismos, y en las muestras de expectoración y líquido pleural, se realizó tinción de Gram y fueron sembradas en medios de cultivo de agar sangre, chocolate y MacConkey e incubadas durante 48 horas a $37^{\circ} \mathrm{C}$. La identificación bacteriana y los estudios de susceptibilidad in vitro se realizaron según métodos convencionales. La sensibilidad a penicilina y cefotaxima fue evaluada midiendo la concentración inhibitoria mínima (CIM) por técnica de epsilometría $\left(\right.$ E-test $\left.^{\mathbb{B}}\right)$ en agar Mueller-Hinton con 5\% de sangre de cordero, siguiendo las recomendaciones del Comité Nacional de Estandarización de los Laboratorios Clínicos (CLSI) ${ }^{18}$. Para el control de calidad se utilizó la cepa de referencia de $S$. pneumoniae ATCC 49619.

\section{Análisis estadístico}

Los resultados fueron expresados como valores promedio \pm desviación estándar para las variables medidas en escala numérica y en porcentaje para las medidas en escala nominal. Las variables cualitativas fueron comparadas mediante la prueba de $\chi^{2}$ o test exacto de Fisher 
y las variables continuas con la prueba t de Student. Las diferencias entre las variables fueron consideradas significativas con un valor de $\mathrm{p}<0,05$.

Se midió la sensibilidad, especificidad, valor predictor positivo y negativo y los odds ratio de los índices pronósticos para predecir los eventos adversos (ingreso a UCI, conexión a ventilador mecánico, complicaciones en el hospital y mortalidad en el seguimiento a 30 días), considerando los puntos de corte definidos por los autores ${ }^{13-15}$. Para comparar la sensibilidad y especificidad de las distintas reglas predictoras se utilizó el test de McNemar. Las curvas receptor operador (ROC) de los índices pronósticos permitieron comparar su capacidad para predecir los distintos eventos adversos. Para ello, se describe el área bajo la curva ROC, el intervalo de confianza de $95 \%$ y valor de $\mathrm{p}$.

\section{Resultados}

Los 151 pacientes incluidos en el estudio tenían una edad promedio de $64 \pm 18$ años, 58\% eran varones, y $75 \%$ tenía una enfermedad pre-existente, especialmente cardiovascular, respiratoria y neurológica crónica. El diagnóstico de la infección neumocóccica fue confirmado mediante cultivo de sangre (n: 73), expectoración (n: 61), líquido pleural (n: 4) y/o antígeno urinario (n: 20). El cuadro clínico y la evolución en el hospital se describen en la Tabla 1.

La mayoría de los pacientes $(96,7 \%)$ fueron tratados con cefalosporinas de segunda o tercera generación asociado, en un tercio de los casos, a macrólidos o fluoroquinolonas. En el estudio de susceptibilidad a antimicrobianos, se identificaron doce cepas resistentes a eritromicina y una cepa resistente a cefalosporinas de tercera generación. No se aislaron cepas de S. pneumoniae resistentes a penicilina $(\mathrm{CIM} \geq 8 \mu \mathrm{g} / \mathrm{mL})$. Todos $10 \mathrm{~s}$ pacientes recibieron tratamiento antimicrobiano apropiado, con cobertura antineumocóccica, en la admisión al hospital.

Treinta y nueve pacientes con NAC grave fueron manejados en la UCI y un tercio requirió conexión a ventilador mecánico. Setenta y tres pacientes (48\%) tuvieron neumonía bacteriémica, lo cual no se asoció a mayor riesgo de complicaciones o muerte en el hospital. Las principales complicaciones en el hospital fueron infecciosas $(17 \%)$, cardiovasculares $(10 \%)$, renales $(9 \%)$ y neurológicas (6\%). La estancia media en el hospital fue $8,5 \pm 6,8$ días y la letalidad fue $11,3 \%$ en el seguimiento a 30 días.

El valor predictor de los índices pronósticos para detectar eventos adversos en pacientes adultos hospitalizados por neumonía neumocóccica se describe en la Tabla 2. Tanto el riesgo de complicaciones y muerte como la proporción de pacientes admitidos a la UCI y

\begin{tabular}{|c|c|}
\hline $\mathrm{n}$ & 151 adultos \\
\hline Edad (años) & $64,2 \pm 18,4(16-92)$ \\
\hline $\operatorname{Sexo}(M / F)$ & $87 / 64(58-42 \%)$ \\
\hline Fumadores & $70(46 \%)$ \\
\hline Índice de Fine: I-II-III-IV-V & $16-17-35-58-25$ (11-11-23-38-17\%) \\
\hline Co-morbilidad & $114(75 \%)$ \\
\hline Enfermedad cardiovascular & $55(36 \%)$ \\
\hline Enfermedad pulmonar obstructiva crónica & $37(25 \%)$ \\
\hline Diabetes mellitus & $27(18 \%)$ \\
\hline Neoplasia & $17(11 \%)$ \\
\hline Enfermedad neurológica crónica & $13(9 \%)$ \\
\hline Asma bronquial & $11(7 \%)$ \\
\hline Enfermedad hepática crónica & $5 \quad(3 \%)$ \\
\hline Insuficiencia renal crónica & 3 (2\%) \\
\hline Lugar de ingreso: Sala-Intermedio-UCI & $87-25-39(58-16-26 \%)$ \\
\hline Uso de ventilación mecánica & $13(9 \%)$ \\
\hline Complicaciones en el hospital & $43(28 \%)$ \\
\hline Neumonía multilobar & 37 (25\%) \\
\hline Efusión pleural & $25(17 \%)$ \\
\hline NAC bacteriémica & $73(48 \%)$ \\
\hline Estadía en el hospital (días) & $8,5 \pm 6,8(1-54)$ \\
\hline Mortalidad en el hospital & $13(8,6 \%)$ \\
\hline Mortalidad a 30 días & $17(11,3 \%)$ \\
\hline \multicolumn{2}{|c|}{$\begin{array}{l}\text { Nota: M: masculino, F: femenino, UCI: Unidad de Cuidados Intensivos, NAC: neumonía adquirida en } \\
\text { la comunidad. Los resultados se expresan como valores promedio } \pm \text { DE o número de casos. }\end{array}$} \\
\hline
\end{tabular}

que requirieron conexión a ventilador mecánico aumentó significativamente en las categorías de alto riesgo de las distintas reglas predictoras. La categoría de bajo riesgo descrita por Fine y cols (clases I-III) permitió discriminar mejor a los pacientes de bajo riesgo de muerte en el seguimiento a 30 días (letalidad: 1,5\%) comparado con los otros índices predictores. En cambio, la categoría de bajo riesgo del INGAC permitió diferenciar con mayor precisión a los pacientes que no requirieron ventilación mecánica.

En la Tabla 3 se compara el área bajo la curva receptor operador (ROC) de las distintas reglas para predecir eventos adversos clínicamente relevantes en el seguimiento en el hospital. El índice de Fine permitió predecir el riesgo de muerte con mayor precisión que el CURB-65 y CRB-65; mientras que el INGAC permitió predecir la conexión a ventilador mecánico con mayor exactitud. La capacidad de predecir la admisión a UCI y el desarrollo de complicaciones en el hospital fue similar para los distintos índices. En la Figura 1 se grafica el área bajo la curva de los distintos índices predictores del riesgo de muerte en el seguimiento a 30 días. 
La sensibilidad, especificidad, valores predictores y odds ratio de los distintos índices predictores de eventos adversos clínicamente relevantes se resumen en la Tabla 4. La estadía en el hospital de los pacientes con neumonía neumocóccica según categorías de riesgo se describe en la Tabla 5. Los pacientes con alto riesgo según los tres

Tabla 2. Valor predictor de eventos adversos clínicamente relevantes de los índices pronósticos en adultos inmunocompetentes hospitalizados por neumonía neumocóccica adquirida en la comunidad

\begin{tabular}{|c|c|c|c|c|}
\hline $\begin{array}{l}\text { Índices } \\
\text { pronósticos }\end{array}$ & $\begin{array}{c}\text { Admisión } \\
\text { a UCI }\end{array}$ & $\begin{array}{c}\text { Ventilación } \\
\text { mecánica }\end{array}$ & $\begin{array}{l}\text { Complicación } \\
\text { en el hospital }\end{array}$ & $\begin{array}{l}\text { Mortalidad a } \\
30 \text { días }\end{array}$ \\
\hline \multicolumn{5}{|l|}{ Índice de Fine ${ }^{13}$} \\
\hline Clases I-II-III & $8 / 68$ & $2 / 68$ & 9/68 & $1 / 68$ \\
\hline Clase IV-V & $31 / 83^{* * *}$ & $11 / 83$ * & $34 / 83^{* * *}$ & $16 / 83^{* * *}$ \\
\hline \multicolumn{5}{|l|}{ CURB- $65^{14}$} \\
\hline $0-2$ puntos & $16 / 95$ & $4 / 95$ & $19 / 95$ & $6 / 95$ \\
\hline$\geq 3$ puntos & $23 / 56^{* *}$ & $9 / 56^{*}$ & $24 / 56^{* *}$ & $11 / 56^{*}$ \\
\hline \multicolumn{5}{|l|}{ CRB-65 ${ }^{17}$} \\
\hline $0-1$ punto & $10 / 73$ & $2 / 73$ & $16 / 73$ & $4 / 73$ \\
\hline$\geq 2$ puntos & $29 / 78^{* *}$ & $11 / 78$ * & $27 / 78$ & $13 / 78^{*}$ \\
\hline \multicolumn{5}{|l|}{ INGAC $^{15}$} \\
\hline $0-19$ puntos & 20/112 & $1 / 112$ & $23 / 112$ & $7 / 112$ \\
\hline$\geq 20$ puntos & $19 / 39 * * *$ & $12 / 39 * * *$ & $20 / 39 * * *$ & $10 / 39^{* *}$ \\
\hline
\end{tabular}

Tabla 3. Capacidad discriminatoria de eventos adversos clínicamente relevantes de los índices pronósticos en adultos inmunocompetentes hospitalizados por neumonía neumocóccica adquirida en la comunidad

\begin{tabular}{lccc} 
Índices pronósticos & Área bajo la curva & IC $95 \%$ & p \\
Índice de Fine & & & \\
Admisión a UCl & 0,669 & $0,588-0,744$ & 0,0001 \\
V. mecánica & 0,789 & $0,715-0,851$ & 0,0001 \\
Complicación & 0,715 & $0,636-0,785$ & 0,0001 \\
Mortalidad & 0,804 & $0,732-0,864$ & 0,0001 \\
CURB-65 & & & \\
Admisión a UCl & 0,709 & $0,630-0,780$ & 0,0001 \\
V. mecánica & 0,737 & $0,660-0,806$ & 0,0001 \\
Complicación & 0,683 & $0,602-0,756$ & 0,0001 \\
Mortalidad & 0,692 & $0,612-0,765$ & 0,0097 \\
CRB-65 & & & \\
Admisión a UCl & 0,692 & $0,612-0,765$ & 0,0001 \\
V. mecánica & 0,691 & $0,611-0,764$ & 0,0011 \\
Complicación & 0,627 & $0,545-0,704$ & 0,0079 \\
Mortalidad & 0,659 & $0,577-0,734$ & 0,0289 \\
INGAC & & & \\
Admisión a UCl & 0,727 & $0,649-0,796$ & 0,0001 \\
V. mecánica & 0,899 & $0,840-0,942$ & 0,0001 \\
Complicación & 0,717 & $0,638-0,787$ & 0,0001 \\
Mortalidad & 0,763 & $0,687-0,828$ & 0,0002 \\
\hline
\end{tabular}

Nota: Comparación del área bajo la curva receptor operador entre predictores: admisión a UCl: no hubo diferencias significativas; ventilación mecánica: INGAC vs CURB-65 o CRB-65 $=p<0,001$; INGAC vs Índice de Fine $=p<0,05$; Índice de Fine vs CRB- $65=p<0,05$; complicaciones en el hospital: INGAC vs CRB- $65=p<0,05$; mortalidad a 30 días: Índice de Fine vs CURB-65 o CRB-65 = $\mathrm{p}<0,05$; INGAC vs CRB-65 $=\mathrm{p}<0,05$. índices pronósticos (IGN, CURB-65 e INGAC) tuvieron una estadía más prolongada en el hospital comparados con las categorías de bajo riesgo $(\mathrm{p}<0,001)$.

\section{Discusión}

El propósito original de los índices pronósticos fue proporcionar ayuda a los médicos clínicos para evaluar la gravedad de los pacientes con neumonía, decidir su lugar de manejo y orientar el tratamiento antimicrobiano empírico $^{19}$. Sin embargo, estos índices fueron diseñados y validados para predecir el riesgo de muerte de los enfermos ${ }^{13-15}$, por lo que se desconoce si entregan además información clínicamente relevante sobre su evolución en el hospital. Evaluar la gravedad y decidir el lugar de manejo del enfermo es una decisión clínica compleja, la cual puede verse dificultada porque los médicos clínicos suelen subestimar, y a veces sobreestimar, la gravedad de la enfermedad ${ }^{20,21}$. Por esta razón, en nuestro estudio evaluamos el valor predictor de cuatro índices de riesgo validados en la literatura científica (índice de Fine, CURB-65, CRB-65, INGAC) $)^{13-15,17}$ con el fin de determinar su capacidad para predecir eventos adversos serios en adultos inmunocompetentes hospitalizados por neumonía neumocóccica. Los principales hallazgos del estudio fueron: Los cuatro índices pronósticos permitieron predecir el riesgo complicaciones y muerte en el seguimiento a 30 días; el índice de Fine (IGN) permitió identificar con mayor precisión a los pacientes con bajo riesgo de muerte; los índices predictores tuvieron distinta sensibilidad y especificidad para detectar los eventos adversos; los índices pronósticos tuvieron elevado valor predictor negativo en la pesquisa de eventos adversos clínicamente relevantes; las categorías de alto riesgo del índice de Fine, CURB-65 e INGAC se asociaron a estancia prolongada en el hospital.

En nuestra serie prospectiva, la letalidad de la neumonía neumocóccica en el hospital $(8,6 \%)$ y en el seguimiento a 30 días $(11,3 \%)$ fue similar a la descrita por otros autores ${ }^{5,22,23}$. Sin embargo, en algunos estudios regionales ${ }^{24-26}$ se han comunicado cifras de letalidad en el adulto hospitalizado por neumonía neumocóccica más elevadas (20-30\%), lo cual pudiera ser parcialmente explicado por la elevada prevalencia de alcoholismo reportado en algunas series ${ }^{24,25}$, diferencias en las co-morbilidades, su gravedad en la admisión al hospital y la disponibilidad de camas de cuidado crítico para el manejo de los enfermos graves. El cuadro clínico, la evolución y pronóstico fueron similares en pacientes con y sin bacteriemia, lo cual había sido descrito por Bordón y cols ${ }^{27}$. Un tercio de los pacientes con neumonía neumocóccica bacteriémica estaban en las categorías de bajo riesgo de Fine y ninguno falleció en el seguimiento a 30 días, experiencia similar a lo descrito por Brandenburg y cols ${ }^{28}$. 
Tabla 4. Sensibilidad, especificidad, valores predictores y odds ratio de las categorías de alto riesgo de los índices pronósticos para pre-

\begin{tabular}{|c|c|c|c|c|c|c|}
\hline Índices pronósticos & $\begin{array}{c}\text { Sensibilidad } \\
\%\end{array}$ & $\begin{array}{c}\text { Especificidad } \\
\%\end{array}$ & $\begin{array}{l}\text { VPP } \\
\%\end{array}$ & $\begin{array}{c}\text { VPN } \\
\%\end{array}$ & $\begin{array}{c}\text { Odds ratio } \\
\%\end{array}$ & $\begin{array}{c}\text { IC } 95 \% \\
\%\end{array}$ \\
\hline \multicolumn{7}{|l|}{ Índice de Fine (IGN) } \\
\hline Admisión a UCl & 79,5 & 53,6 & 37,4 & 88,2 & 4,5 & $1,9-10,6$ \\
\hline V. mecánica & 84,6 & 47,8 & 13,3 & 97,1 & 5,0 & $1,1-23,6$ \\
\hline Complicación & 79,1 & 54,6 & 41,0 & 86,8 & 4,5 & $2,0-10,4$ \\
\hline Mortalidad & 94,1 & 50,0 & 19,3 & 98,5 & 16,0 & $2,0-124$ \\
\hline \multicolumn{7}{|l|}{ CURB-65 } \\
\hline Admisión a UCI & 59,0 & 70,5 & 41,1 & 83,2 & 3,4 & $1,6-7,3$ \\
\hline V. mecánica & 69,2 & 65,9 & 16,1 & 95,8 & 4,4 & $1,3-14,9$ \\
\hline Complicación & 55,8 & 70,4 & 42,9 & 80,0 & 3,0 & $1,5-6,2$ \\
\hline Mortalidad & 64,7 & 66,4 & 19,6 & 93,7 & 3,6 & $1,3-10,4$ \\
\hline \multicolumn{7}{|l|}{ CRB-65 } \\
\hline Admisión a UCl & 74,4 & 56,3 & 37,2 & 86,3 & 3,7 & $1,7-8,3$ \\
\hline V. mecánica & 84,6 & 51,5 & 14,1 & 97,3 & 5,8 & $1,2-27,2$ \\
\hline Complicación & 63,0 & 52,8 & 34,6 & 78,1 & 1,9 & $0,9-3,8$ \\
\hline Mortalidad & 76,5 & 51,5 & 16,7 & 94,5 & 3,5 & $1,1-11,1$ \\
\hline \multicolumn{7}{|l|}{ INGAC } \\
\hline Admisión a UCl & 48,7 & 82,1 & 48,7 & 82,1 & 4,4 & $2,0-9,6$ \\
\hline V. mecánica & 92,3 & 80,4 & 30,8 & 99,1 & 49,3 & $6,1-396$ \\
\hline Complicación & 46,5 & 82,4 & 51,3 & 79,5 & 4,1 & $1,9-8,9$ \\
\hline Mortalidad & 58,8 & 78,4 & 25,6 & 93,8 & 5,2 & $1,8-14,8$ \\
\hline
\end{tabular}

Nota: VPP: Valor predictor positivo, VPN: Valor predictor negativo, IC 95\%: intervalo de confianza de 95\%, UCl: Unidad de cuidado intensivo, V. mecánica: Ventilación mecánica.

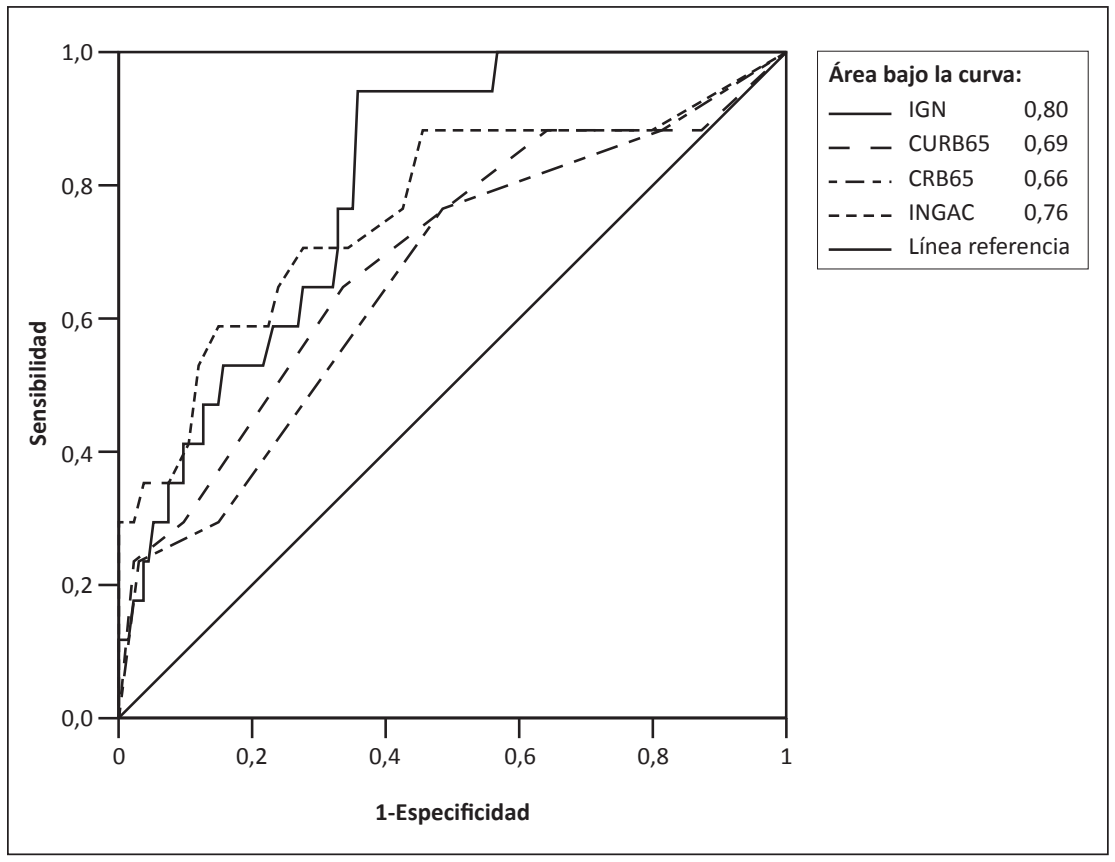

Tabla 5. Estadía en el hospital de los pacientes con neumonía neumocóccica según categorías de riesgo de los índices predictores de mortalidad

\begin{tabular}{lccc|}
$\begin{array}{l}\text { Índices } \\
\text { pronósticos }\end{array}$ & Bajo riesgo & Riesgo elevado & p \\
\hline IGN & $6,31 \pm 4,35$ & $10,36 \pm 7,94$ & 0,0002 \\
\hline CURB-65 & $6,98 \pm 5,05$ & $11,18 \pm 8,57$ & 0,0002 \\
\hline CRB-65 & $7,42 \pm 5,32$ & $9,58 \pm 7,92$ & 0,0526 \\
\hline INGAC & $6,91 \pm 4,05$ & $13,21 \pm 10,37$ & 0,0001 \\
\hline \multicolumn{4}{l}{$\begin{array}{l}\text { Nota: Los resultados se expresan como valores promedio } \\
\text { la estadía en el hospital (días). }\end{array}$} \\
\hline
\end{tabular}

Figura 1. Áreas bajo la curva receptor operador de los índices predictores de mortalidad en adultos hospitalizados por neumonía neumocóccica adquirida en la comunidad. 
Los cuatros índices pronósticos permitieron identificar a los pacientes con alto riesgo de muerte en el seguimiento a 30 días $^{13-15,17,19}$. Sin embargo, el índice de Fine fue más sensible y el INGAC fue más específico para predecir el riesgo de muerte comparado con el CURB-65 y CRB-65. Las categorías de bajo riesgo descritas por Fine y cols (clases I a III) detectaron a los pacientes hospitalizados por neumonía neumocóccica con bajo riesgo de muerte (menor de 2\%); mientras que las otras reglas predictoras fueron menos sensibles y específicas para definir al grupo de bajo riesgo (Tabla 2).

Varios estudios han demostrado que los índices de Fine y CURB-65 no constituyen la herramienta ideal para predecir el ingreso a $\mathrm{UCI}^{29,30}$, siendo mejor predictor el índice INGAC $^{31}$. En nuestro estudio, el poder discriminatorio de los diferentes índices para predecir la admisión a UCI fue similar (Tabla 3). Por este motivo, la Sociedades de Tórax y Enfermedades Infecciosas de Estados Unidos han propuesto una nueva regla predictora que aún no ha sido evaluada y validada en grandes poblaciones ${ }^{10}$.

Los índices pronósticos validados en la literatura científica (IGN, CURB-65 e INGAC) permiten estratificar apropiadamente a los pacientes que requieren ventilación mecánica ${ }^{29,32}$. Similar a lo descrito por España y cols ${ }^{31}$, el INGAC fue más sensible y específico en la pesquisa de pacientes con falla respiratoria grave que requirieron conexión a ventilador mecánico. Sin embargo, estos índices pronósticos no fueron muy precisos en la detección de los pacientes con neumonía que desarrollaron complicaciones durante su estadía en el hospital.

Los índices pronósticos tuvieron un elevado valor predictor negativo, por lo cual en los pacientes de las categorías de bajo riesgo fue improbable la aparición de eventos adversos clínicamente significativos, especialmente la necesidad de ventilación mecánica y el riesgo de muerte en el seguimiento a 30 días. España y cols ${ }^{31}$, destacaron el elevado valor predictor negativo de los índices de Fine, CURB-65 e INGAC para los siguientes eventos adversos: admisión a UCI, conexión a ventilador mecánico, desarrollo de sepsis y fracaso de tratamiento empírico.

Las reglas de predicción de la Sociedad Británica de Tórax (CURB-65) e INGAC son más sencillas y fáciles de implementar en la atención ambulatoria, ya que requieren evaluar cinco u ocho variables clínicas y de laboratorio, respectivamente, a diferencia del índice de Fine que considera 20 variables. Sin embargo, el poder discriminatorio del CURB-65 se vería limitado en población senescente por los puntos de corte de la presión diastólica $(\leq 60 \mathrm{mmHg})$ y nitrógeno ureico $(>20$ $\mathrm{mg} / \mathrm{dL}$ ), anomalías frecuentes de observar en pacientes ancianos ${ }^{33}$. Además, este índice no incluye el estado de oxigenación del paciente, lo cual debe ser evaluado en forma sistemática por los médicos en los servicios de urgencia y corregido en forma oportuna si se pesquisa anomalías del intercambio gaseoso.

Similar a lo descrito por otros autores, los tres índices pronósticos (IGN, CURB-65 e INGAC) se relacionaron significativamente con la permanencia en el hospital ${ }^{29,31,32}$. De hecho, las categorías de alto riesgo se asociaron a estancia prolongada en el hospital (Tabla 5).

En conclusión, los índices de gravedad propuestos en las guías clínicas de neumonía ${ }^{10-12}$ (IGN, CURB-65), y el IGNAC recientemente descrito, permiten predecir el riesgo de complicaciones y muerte en adultos hospitalizados con neumonía neumocóccica adquirida en la comunidad. Sin embargo, los índices pronósticos difieren en su capacidad discriminatoria y precisión para predecir los distintos eventos adversos clínicamente relevantes en la evolución, que serían de utilidad para los médicos clínicos en el manejo de los pacientes hospitalizados por NAC.

\section{Resumen}

Streptococcus pneumoniae es el principal agente causal de la neumonía adquirida en la comunidad. Objetivos: Examinar el poder discriminativo de tres índices pronósticos en la predicción de eventos adversos clínicamente relevantes en pacientes hospitalizados por neumonía neumocóccica adquirida en la comunidad. Métodos: Evaluamos el índice de gravedad de la neumonía (IGN), CURB-65 y el índice de neumonía grave adquirida en la comunidad (INGAC) en una cohorte de 151 adultos inmunocompetentes hospitalizados por neumonía neumocóccica. Los eventos adversos examinados fueron la admisión a UCI, necesidad de ventilación mecánica, complicaciones en el hospital y mortalidad a 30 días. Las reglas predictoras fueron comparadas en base a su sensibilidad, especificidad y área bajo la curva receptor operador. Resultados: Se evaluaron 151 pacientes (64 \pm 18 años), 58\% varones, $75 \%$ tenía co-morbilidad, $26 \%$ fueron admitidos a la UCI y $9 \%$ requirieron ventilación mecánica. La tasa de eventos adversos fue más elevada y la estadía en el hospital más prolongada en las categorías de alto riesgo de los tres índices predictores. Los tres índices permitieron, a su vez, predecir el riesgo de complicaciones y muerte en el seguimiento a 30 días. El IGN fue más sensible y el INGAC más específico en la pesquisa de complicaciones en el hospital y en predecir el riesgo de muerte. El INGAC fue más sensible y específico en predecir el uso de ventilación mecánica. El CURB-65 tuvo menor poder discriminatorio comparado con el IGN e INGAC. Conclusión: Los índices pronósticos validados en la literatura médica permiten predecir el riesgo de complicaciones y muerte en el adulto hospitalizado por neumonía neumocóccica. Sin embargo, difieren en su sensibilidad, especificidad y poder discriminatorio de los distintos eventos adversos. 


\section{Referencias}

1.- Norby S R, Pope K A. Pneumococcal pneumonia. J Infect 1979; 1: 109-20.

2.- Afessa B, Greaves W L, Frederick W R. Pneumococcal bacteremia in adults: a 14-year experience in an inner-city university hospital. Clin Infect Dis 1995; 21: 345-51.

3.- Fine M J, Smith M A, Carson C A, Mutha S S, Sankey S S, Weissfeld L A, et al. Prognosis and outcomes of patients with community-acquired pneumonia. A meta-analysis. JAMA 1996; 275 : 134-41.

4.- Burman L A, Norrby R, Trollfors B. Invasive pneumococcal infections: incidence, predisposing factors, and prognosis. Rev Infect Dis 1985; 7: 133-42.

5.- Ortqvist A, Grepe A, Julander I, Kalin M. Bacteremic pneumococcal pneumonia in Sweden: clinical course and outcome and comparison with non-bacteremic pneumococcal and mycoplasmal pneumonias. Scand J Infect Dis 1988; 20: 163-71.

6.- Austrian R, Gold J. Pneumococcal bacteremia with especial reference to bacteremic pneumococcal pneumonia. Ann Intern Med 1964; 60: 759-76.

7.- Frei C R, Restrepo M I, Mortensen E M, Burgess DS. Impact of guideline-concordant empiric antibiotic therapy in communityacquired pneumonia. Am J Med 2006; 119: 865-71.

8.- Reyes S, Martínez R, Cremades M J, Martínez E, Soler J J, Menéndez R. Empiric treatment in hospitalized community-acquired pneumonia. Impact on mortality, length of stay and re-admission. Respir Med 2007;101: 1909-15.

9.- Martínez R, Reyes S, Lorenzo M J, Menéndez R. Impact of guidelines on outcome: the evidence. Semin Respir Crit Care Med 2009; 30: 172-8.

10.- Mandell L A, Wunderink R G, Anzueto A, Bartlett J G, Campbell G D, Dean N C, et al. Infectious Diseases Society of America/ American Thoracic Society Consensus Guidelines on the management of communityacquired pneumonia in adults. Clin Infect Dis 2007; 44 (Suppl 2): S27-S72.

11.- Lim W S, Baudouin S V, George R C, Hill A T, Jamieson C, Le Jeune I, et al; Pneumonia Guidelines Committee of the BTS Standards of Care Committee. The British Thoracic Society Guidelines for the management of communityacquired pneumonia in adults: update 2009. Thorax 2009; 64 (Suppl 3):1-55.

12.- Saldías F, Pavié J. Consenso Chileno de Neumonía Comunitaria. Evaluación de la gravedad en la neumonía del adulto adquirida en la comunidad. Rev Chil Infectol 2005; 22
(Supl 1): S39-S45.

13.- Fine M J, Auble T E, Yealy D M, Hanusa B H, Weissfeld L A, Singer D E, et al. A prediction rule to identify low-risk patients with community-acquired pneumonia. N Engl J Med 1997; 336: 243-50.

14.- Lim W S, Van Der Eerden M M, Laing R, Boersma W G, Karalus N, Town G I, et al. Defining community acquired pneumonia severity on presentation to hospital: an international derivation and validation study. Thorax 2003; 58: 377-82.

15.- España P P, Capelastegui A, Gorordo I, Esteban C, Oribe M, Ortega M, et al. Development and validation of a clinical prediction rule for severe community-acquired pneumonia. Am J Respir Crit Care Med 2006; 174: $1249-56$.

16.- Fang G D, Fine M, Orloff J, Arisumi D, Yu V L, Kapoor W, et al. New emerging etiologies for community-acquired pneumonia with implications for therapy. A prospective multicenter study of 359 cases. Medicine (Baltimore) 1990; 69: 307-16.

17.- Bauer T T, Ewig S, Marre R, Suttorp N, Welte T; The CAPNETZ Study Group. CRB-65 predicts death from community-acquired pneumonia. J Intern Med 2006; 260: 93-101.

18.- Clinical and Laboratory Standards Institute. Performance standards for antimicrobial susceptibility testing: $18^{\text {th }}$ informational supplement. CLSI document M100-S18. Wayne, PA: Clinical and Laboratory Standards Institute, 2008.

19.- Niederman M S. Making sense of scoring systems in community acquired pneumonia. Respirology 2009; 14: 327-35.

20.- Fine M J, Hough L J, Medsger A R, Li Y H, Ricci E M, Singer D E, et al. The hospital admission decision for patients with community-acquired pneumonia. Results from the Pneumonia Patient Outcomes Research Team cohort study. Arch Intern Med 1997; 157 : 36-44.

21.- Neill A M, Martin I R, Weir R, Anderson R, Chereshsky A, Epton M J, et al. Community acquired pneumonia: aetiology and usefulness of severity criteria on admission. Thorax 1996; 51: 1010-6.

22.- Díaz A, Torres C, Flores L J, García P, Saldías F. Community acquired pneumococcal pneumonia in hospitalized adult patients. Rev Med Chile 2003; 131: 505-14.

23.- Frankel R E, Virata M, Hardalo C, Altice F L, Friedland G. Invasive pneumococcal disease: clinical features, serotypes, and antimicrobial resistance patterns in cases involving patients with and without human immunodeficiency virus infection. Clin Infect Dis 1996; 23 577-84.
24.- Noriega L M, González P, Canals C, Michaud P. Septicemia por Streptococcus pneumoniae. Análisis de 40 casos. Rev Med Chile 1994; 122: 1385-92.

25.- Rioseco M L, Riquelme R. Neumonía neumocócica bacterémica en 45 adultos inmunocompetentes hospitalizados. Cuadro clínico y factores pronósticos. Rev Med Chile 2004; 132: 588-94

26.- Mella S, Blamey R, Paiva O, Yáñez J, Riedel G, Aylwin M, et al. Impacto de la evaluación infectológica en el uso racional de antimicrobianos en pacientes con bacteriemia por Streptococcus pneumoniae. Rev Chil Infectol 2007; 24: 264-9.

27.- Bordón J, Peyrani P, Brock G N, Blasi F, Rello J, File T, et al; CAPO Study Group. The presence of pneumococcal bacteremia does not influence clinical outcomes in patients with community-acquired pneumonia: results from the Community-Acquired Pneumonia Organization (CAPO) International Cohort study. Chest 2008; 133: 618-24.

28.- Brandenburg J A, Marrie T J, Coley C M, Singer D E, Obrosky D S, Kapoor W N, et al. Clinical presentation, processes and outcomes of care for patients with pneumococcal pneumonia. J Gen Intern Med 2000; 15: 638-46.

29.- Capelastegui A, España P P, Quintana J M, Areitio I, Gorordo I, Egurrola M, et al. Validation of a predictive rule for the management of community-acquired pneumonia. Eur Respir J 2006; 27 : 151-7.

30.- Man S Y, Lee N, Ip M, Antonio G E, Chau S S, Mak P, et al. Prospective comparison of three predictive rules for assessing severity of community-acquired pneumonia in Hong Kong. Thorax 2007; 62: 348-53.

31.- España P P, Capelastegui A, Quintana J, Diez R, Gorordo I, Bilbao A, et al. Prospective comparison of severity scores for predicting clinically relevant outcomes for patients hospitalized with community-acquired pneumonia. Chest 2009; 135: 1572-9.

32.- Buising K L, Thursky K A, Black J F, MacGregor L, Street A C, Kennedy M P, et al. A prospective comparison of severity scores for identifying patients with severe communityacquired pneumonia: reconsidering what is meant by severe pneumonia. Thorax 2006; 61: 419-24.

33.- Myint P K, Kamath A V, Vowler S L, Maisey D N, Harrison B D. The CURB (confusion, urea, respiratory rate and blood pressure) criteria in community-acquired pneumonia (CAP) in hospitalised elderly patients age 65 years and over: a prospective observational cohort study. Age Ageing 2005; 34: 75-7. 